\title{
NONCOMMUTATIVE GEOMETRY AND THE STANDARD MODEL WITH NEUTRINO MIXING
}

\author{
ALAIN CONNES
}

\begin{abstract}
We show that allowing the metric dimension of a space to be independent of its KO-dimension and turning the finite noncommutative geometry $\mathrm{F}$ - whose product with classical 4-dimensional space-time gives the standard model coupled with gravity-into a space of KO-dimension 6 by changing the grading on the antiparticle sector into its opposite, allows to solve three problems of the previous noncommutative geometry interpretation of the standard model of particle physics: The finite geometry $\mathrm{F}$ is no longer put in "by hand" but a conceptual understanding of its structure and a classification of its metrics is given. The fermion doubling problem in the fermionic part of the action is resolved. The spectral action of our joint work with Chamseddine now automatically generates the full standard model coupled with gravity with neutrino mixing and see-saw mechanism for neutrino masses. The predictions of the Weinberg angle and the Higgs scattering parameter at unification scale are the same as in our joint work but we also find a mass relation (to be imposed at unification scale).
\end{abstract}

\section{INTRODUCTION}

We showed some time ago ( $c f$. [6]) how to interpret the Lagrangian of the standard model in terms of noncommutative geometry. This interpretation was based on the extension of the Yang-Mills functional to the algebraic framework of NCG. In [6] the color degrees of freedom were still added in an artificial manner and the action functional was obtained by analogy with the classical gauge theories. In our joint work with A. Chamseddine [1], 2] and in $[8$ we showed how to incorporate the color naturally and more importantly how to obtain the bosonic part of the standard model action coupled to gravity from a very general spectral action principle. We call it a principle since it is based on the very general idea that a refined notion of geometry (suitable in particular to deal with spaces whose coordinates do not commute) is obtained by focussing not on the traditional $g_{\mu \nu}$ but on the Dirac operator $D$. As it turns out this way of defining a geometry by specifying the Dirac operator is meaningful both in mathematical terms (where the Dirac operator specifies the fundamental class in KO-homology) and in physics terms (where, modulo a chiral gauge transformation, the Dirac operator is the inverse of the Euclidean propagator of fermions). The spectral action principle then asserts that $D$ is all that is needed to define the bosonic part of the action. Moreover since disjoint union of spaces correspond to direct sums of the Dirac operators, a simple additivity requirement of the action functional shows that it has to be of the form

$$
S=\operatorname{Tr}(f(D / \Lambda))
$$


where $f$ is an even function of the real variable and $\Lambda$ a parameter fixing the mass scale. In fact the choice of the test function $f$ only plays a small role since when expanded in inverse powers of $\Lambda$ the action $S$ only depends on the first moments $\int f(u) u^{k-1} d u$ and the Taylor expansion of $f$ at 0 .

Not surprisingly the gravitational Einstein action appears naturally in the expansion of $S$, a point which is reminiscent of the idea of induced gravity. Moreover in the presence of gauge fields $A$ the operator $D$ gets modified (replacing derivatives by covariant derivatives) to $D_{A}$ and the Yang-Mills action functional $\mathrm{YM}(A)$ appears, in its Euclidean form and with the correct sign if $f \geq 0$, in the coefficient of $\Lambda^{0}$ in the spectral action (11) for the operator $D_{A}$. The simple idea developed in [1, then, is that one should understand the modification $D \rightarrow D_{A}$ coming from the presence of gauge fields as a slight change in the metric, while the action principle (11) which is in essence purely gravitational delivers when applied to $D_{A}$ the combined Einstein-Yang-Mills action. To keep track of the "change of metric" coming from the gauge fields one needs to enhance the algebra of coordinates on the manifold $M$ to the algebra of matrix valued functions on $M$ which encodes the gauge group as its group of inner automorphisms. We refer to section 2 of [2] for the case of an $\mathrm{SU}(n)$ theory.

The advantage in passing to noncommutative algebras of coordinates $\mathcal{A}$ is that their automorphism group $\operatorname{Aut}(\mathcal{A})$ admits a decomposition

$$
1 \rightarrow \operatorname{Inn}(\mathcal{A}) \rightarrow \operatorname{Aut}(\mathcal{A}) \rightarrow \operatorname{Out}(\mathcal{A}) \rightarrow 1
$$

into inner and outer parts, which fits very well with the physics distinction between the internal symmetries $g \in \mathcal{G}$ and the others, i.e. the exact sequence governing the structure of the symmetry group $\mathcal{U}$ of the combined Lagrangian of gravity and matter,

$$
1 \rightarrow \mathcal{G} \rightarrow \mathcal{U} \rightarrow \operatorname{Diff}(M) \rightarrow 1
$$

Moreover a similar decomposition into an "inner" piece and an outer one holds at the level of the noncommutative metric i.e. of the Dirac operator. Thus in the noncommutative world, the metrics (encoded by $D$ ) admit natural "inner fluctuations" which come directly from the self Morita equivalence $\mathcal{A} \sim \mathcal{A}$ and are encoded by gauge potentials i.e. operators of the form

$$
A=\sum a_{j}\left[D, b_{j}\right], \quad a_{j}, b_{j} \in \mathcal{A}, \quad A=A^{*}
$$

The main result of our joint work [1], 2] is that, when applied to the inner fluctuations of the product geometry $M \times F$ the spectral action gives the standard model coupled with gravity. Here $M$ is a Riemannian compact spin 4-manifold, the standard model coupled with gravity is in the Euclidean form, and the geometry of the finite space $F$ is encoded (as in the general framework of NCG) by a spectral triple $\left(\mathcal{A}_{F}, \mathcal{H}_{F}, D_{F}\right)$ i.e. by a Hilbert space $\mathcal{H}_{F}$, a representation of the algebra of coordinates $\mathcal{A}_{F}$, and the inverse line element $D_{F}$. Besides a $\mathbb{Z} / 2$ grading $\gamma$ this spectral triple has a crucial piece of structure: a real structure (cf. [7]) i.e. an antilinear isometry of $\mathcal{H}$ of square \pm 1 with simple algebraic rules and whose dimension, called the KO-dimension is well defined modulo 8 from the signs involved in the algebraic rules ( $c f$. Appendix 7).

For the noncommutative geometry $F$ used in 2 to obtain the standard model coupled to gravity, all the ingredients are finite dimensional. The algebra $\mathcal{A}_{F}=\mathbb{C} \oplus \mathbb{H} \oplus M_{3}(\mathbb{C})$ (i.e. the direct sum of the algebras $\mathbb{C}$ of complex numbers, $\mathbb{H}$ of quaternions, and $M_{3}(\mathbb{C})$ of $3 \times 3$ matrices) encodes the gauge group. The Hilbert space $\mathcal{H}_{F}$ is of dimension $90^{1}$ and encodes

\footnotetext{
${ }^{1}$ It is 96 in the model described below
} 
the elementary quarks and leptons. The operator $D_{F}$ encodes those free parameters of the standard model related to the Yukawa couplings.

For $M$ the spectral triple is given by the representation of the algebra of smooth functions acting by multiplication in the Hilbert space $L^{2}(M, S)$ of square integrable spinors, the grading is given by $\gamma_{5}$ and the real structure $J_{M}$ is given by charge conjugation.

While it is certainly remarkable to obtain the standard model action from simple geometric principles the above work has several shortcomings:

(1) The finite geometry $F$ is put in "by hand" with no conceptual understanding of the representation of $\mathcal{A}_{F}$ in $\mathcal{H}_{F}$.

(2) There is a fermion doubling problem (cf. [15]) in the fermionic part of the action.

(3) It does not incorporate the neutrino mixing and see-saw mechanism for neutrino masses.

We shall show in this note how to solve these three problems (the first only partly since the number of generations is put by hand) simply by keeping the distinction between the following two notions of dimension of a noncommutative space,

- The metric dimension

- The KO-dimension

The metric dimension manifests itself by the growth of the spectrum of the Dirac operator. As far as space-time goes it appears that the situation of interest will be the 4-dimensional one. In particular the metric dimension of the finite geometry $F$ will be zero.

The $K O$-dimension is only well defined modulo 8 and it takes into account both the $\mathbb{Z} / 2$ grading $\gamma$ of $\mathcal{H}$ as well as the real structure $J$ ( $c f$. Appendix 7 ). The real surprise is that in order for things to work the only needed change (besides the easy addition of a right handed neutrino) is to change the $\mathbb{Z} / 2$ grading of the finite geometry $F$ to its opposite in the "antiparticle" sector. It is only thanks to this that the Fermion doubling problem pointed out in 15. can be successfully handled. Moreover it will automatically generate the full standard model i.e. the model with neutrino mixing and the see-saw mechanism as follows from the full classification of Dirac operators: Theorem 2.7.

When one looks at the table (17.2) of Appendix 7 giving the $K O$-dimension of the finite space $F$ one then finds that its $\mathrm{KO}$-dimension is now equal to 6 modulo $8(!)$. As a result we see that the $K O$-dimension of the product space $M \times F$ is in fact equal to $10 \sim 2$ modulo 8 . Of course the above 10 is very reminiscent of string theory, in which the finite space $F$ might be a good candidate for an "effective" compactification at least for low energies2. But 10 is also 2 modulo 8 which might be related to the observations of [14 about gravity.

It is also remarkable that the noncommutative spheres arising from quantum groups, such as the Podleś spheres already exhibit the situation where the metric dimension (0 in that case) is distinct from the $\mathrm{KO}$-dimension ( 2 in that case) as pointed out in [10].

We have gathered the definitions of the basic notions of noncommutative geometry: spectral triples, real structure and inner fluctuations, in the Appendix 7. We shall often refer to these basic notions in the text and urge the reader unfamiliar with these to start by a brief look at the appendix.

\footnotetext{
${ }^{2}$ Note however that we are dealing with the standard model, not its supersymmetrized version.
} 


\section{The Finite NON COMmUtative GeOMETRY $F$}

In this section we shall first describe in a conceptual manner the representation of $\mathcal{A}_{F}$ in $\mathcal{H}_{F}$ and classify the Dirac operators $D_{F}$. The only small nuance with [8] is that we incorporate a right handed neutrino $\nu_{R}$ and change the $\mathbb{Z} / 2$ grading in the antiparticle sector to its opposite. This, innocent as it looks, allows for a better conceptual understanding of the representation of $\mathcal{A}_{F}$ in $\mathcal{H}_{F}$ and also will completely alter the classification of Dirac operators (Theorem 2.7).

\subsection{The representation of $\mathcal{A}_{F}$ in $\mathcal{H}_{F}$.}

We start from the involutive algebra (with $\mathbb{H}$ the quaternions with involution $q \rightarrow \bar{q}$ )

$$
\mathcal{A}_{L R}=\mathbb{C} \oplus \mathbb{H}_{L} \oplus \mathbb{H}_{R} \oplus M_{3}(\mathbb{C})
$$

We are looking for a natural representation $\left(\mathcal{A}_{L R}, \mathcal{H}_{F}, J_{F}, \gamma_{F}\right)$ fulfilling definition 7.2 of Appendix 7 in dimension 6 modulo 8. The commutation relation (44) of definition 7.2 shows that there is an underlying structure of $\mathcal{A}_{L R}$-bimodule on $\mathcal{H}_{F}$ and we shall use that structure as a guide. One uses the bimodule structure to define $\operatorname{Ad}(u)$, for $u \in \mathcal{A}$ unitary, by

$$
\operatorname{Ad}(u) \xi=u \xi u^{*}
$$

Definition 2.1. Let $\mathcal{M}$ be an $\mathcal{A}_{L R}$-bimodule. Then $\mathcal{M}$ is odd iff the adjoint action (3) of $s=(1,-1,-1,1)$ fulfills $\operatorname{Ad}(s)=-1$.

Such a bimodule is a representation of the reduction of $\mathcal{A}_{L R} \otimes_{\mathbb{R}} \mathcal{A}_{L R}^{0}$ by the projection $\frac{1}{2}\left(1-s \otimes s^{0}\right)$. This subalgebra is an algebra over $\mathbb{C}$ and we restrict to complex representations. One defines the contragredient bimodule of a bimodule $\mathcal{M}$ as the complex conjugate space

$$
\mathcal{M}^{0}=\{\bar{\xi} ; \xi \in \mathcal{M}\}, \quad a \bar{\xi} b=\overline{b^{*} \xi a^{*}}, \quad \forall a, b \in \mathcal{A}_{L R}
$$

We can now give the following characterization of the $\mathcal{A}_{L R}$-bimodule $\mathcal{M}_{F}$ and the real structure $J_{F}$ for one generation.

Proposition 2.2. $\quad$ - The $\mathcal{A}_{L R}$-bimodule $\mathcal{M}_{F}$ is the direct sum of all inequivalent irreducible odd $\mathcal{A}_{L R}$-bimodules.

- The dimension of $\mathcal{M}_{F}$ is 32 .

- The real structure $J_{F}$ is given by the isomorphism with the contragredient bimodule.

We define the $\mathbb{Z} / 2$-grading $\gamma_{F}$ by

$$
\gamma_{F}=c-J_{F} c J_{F}, \quad c=(0,-1,1,0) \in \mathcal{A}_{L R}
$$

One then checks that the following holds

$$
J_{F}^{2}=1, \quad J_{F} \gamma_{F}=-\gamma_{F} J_{F}
$$

which together with the commutation of $J_{F}$ with the Dirac operators, is characteristic of $K O$-dimension equal to 6 modulo 8 ( $c f$. Appendix 7, definition 7.2).

The equality $\iota(\lambda, q, m)=(\lambda, q, \lambda, m)$ defines a homomorphism $\iota$ of involutive algebras from $\mathcal{A}_{F}$ to $\mathcal{A}_{L R}$ so that we view $\mathcal{A}_{F}$ as a subalgebra of $\mathcal{A}_{L R}$.

Definition 2.3. The real representation $\left(\mathcal{A}_{F}, \mathcal{H}_{F}, J_{F}, \gamma_{F}\right)$ is the restriction to $\mathcal{A}_{F} \subset \mathcal{A}_{L R}$ of the direct sum $\mathcal{M}_{F} \otimes \mathbb{C}^{3}$ of three copies of $\mathcal{M}_{F}$.

It has dimension $32 \times 3=96$, needless to say this 3 is the number of generations and it is put in by hand here. 


\subsection{The unimodular unitary group $\mathrm{SU}\left(\mathcal{A}_{F}\right)$.}

Using the action of $\mathcal{A}_{F}$ in $\mathcal{H}_{F}$ one defines the unimodular subgroup $\mathrm{SU}\left(\mathcal{A}_{F}\right)$ of the unitary group $\mathrm{U}\left(\mathcal{A}_{F}\right)=\left\{u \in \mathcal{A}_{F}, u u^{*}=u^{*} u=1\right\}$ as follows,

Definition 2.4. We let $\mathrm{SU}\left(\mathcal{A}_{F}\right)$ be the subgroup of $\mathrm{U}\left(\mathcal{A}_{F}\right)$ defined by

$$
\mathrm{SU}\left(\mathcal{A}_{F}\right)=\left\{u \in \mathrm{U}\left(\mathcal{A}_{F}\right): \operatorname{Det}(u)=1\right\}
$$

where $\operatorname{Det}(u)$ is the determinant of the action of $u$ in $\mathcal{H}_{F}$.

One obtains both the standard model gauge group and its action on fermions from the adjoint action of $\mathrm{SU}\left(\mathcal{A}_{F}\right)$ in the following way:

Proposition 2.5. (1) The group $\mathrm{SU}\left(\mathcal{A}_{F}\right)$ is, up to an abelian finite group,

$$
\mathrm{SU}\left(\mathcal{A}_{F}\right) \sim \mathrm{U}(1) \times \mathrm{SU}(2) \times \mathrm{SU}(3)
$$

(2) The adjoint action $u \rightarrow \operatorname{Ad}(u)$ (cf. (3) ) of $\mathrm{SU}\left(\mathcal{A}_{F}\right)$ in $\mathcal{H}_{F}$ coincides with the standard model action on elementary quarks and leptons.

To see what happens we first have to label the basis of the bimodule $\mathcal{M}_{F}$. We use the following idempotents in $\mathcal{A}_{L R}$,

$$
e_{\ell}=(1,0,0,0), \quad e_{L}=(0,1,0,0), \quad e_{R}=(0,0,1,0), \quad e_{q}=(0,0,0,1) .
$$

The reduced algebras are respectively $\mathbb{C}, \mathbb{H}_{L}, \mathbb{H}_{R}, M_{3}(\mathbb{C})$. One has $\sum e_{j}=1$ and similarly in the algebra $\mathcal{A}_{L R} \otimes \mathcal{A}_{L R}^{0}$ one has

$$
\sum e_{j} \otimes e_{k}^{0}=1
$$

Using the action of $\mathcal{A}_{L R} \otimes \mathcal{A}_{L R}^{0}$ associated to the bimodule structure of $\mathcal{M}$ thus gives a decomposition of the form

$$
\mathcal{M}=\sum e_{j} \mathcal{M} e_{k}
$$

Since $\mathcal{M}$ is odd this decomposition can be written as

$$
\mathcal{M}=\sum e_{j} \mathcal{M} e_{K}+\sum e_{J} \mathcal{M} e_{k}
$$

Let us consider the term $e_{L} \mathcal{M} e_{\ell}$. It is a $\mathbb{H}_{L^{-}}$left, $\mathbb{C}$-right module. Thus it is a multiple of the only irreducible representation $\pi_{L}$ of $\mathbb{H}_{L}$ which is two dimensional. The action of $\mathbb{C}$ is given by the scalar action. Let us consider the term $e_{L} \mathcal{M} e_{q}$. It is a $\mathbb{H}_{L}$-left, $M_{3}(\mathbb{C})$-right module. Since the algebra $\mathbb{H}_{L} \otimes_{\mathbb{R}} M_{3}(\mathbb{C})$ is $M_{6}(\mathbb{C})$ we see that all such bimodules are multiples of the bimodule $\pi_{L}^{3}$ given for the left action of $\mathbb{H}_{L}$ as the direct sum of three copies of $\pi_{L}$ and with the obvious right action of $M_{3}(\mathbb{C})$ permuting the three copies. Exactly the same holds for the bimodules $e_{R} \mathcal{M} e_{\ell}$ and $e_{R} \mathcal{M} e_{q}$, which are respectively multiples of $\pi_{R}$ and of $\pi_{R}^{3}$. Similar results hold switching the left and right actions i.e. by passing to the contragredient bimodule of $\mathcal{M}$. We thus see that the sum of the irreducible odd bimodules is given by

$$
\mathcal{M}_{F}=\left(\pi_{L} \oplus \pi_{R} \oplus \pi_{R}^{3} \oplus \pi_{L}^{3}\right) \oplus\left(\pi_{L} \oplus \pi_{R} \oplus \pi_{R}^{3} \oplus \pi_{L}^{3}\right)^{0}
$$

This $\mathcal{A}_{L R}$-bimodule $\mathcal{M}_{F}$ is of dimension $2 \cdot(2+2+2 \times 3+2 \times 3)=32$ and the adjoint action gives the gauge action of the standard model for one generation, with the following labels for the basis elements of $\mathcal{M}_{F}$,

$$
\left(\begin{array}{ll}
\nu_{L} & \nu_{R} \\
e_{L} & e_{R}
\end{array}\right)
$$


for the term $\pi_{L} \oplus \pi_{R}$,

$$
\left(\begin{array}{ll}
u_{L}^{j} & u_{R}^{j} \\
d_{L}^{j} & d_{R}^{j}
\end{array}\right)
$$

for the term $\pi_{R}^{3} \oplus \pi_{L}^{3}$ ( with color indices $j$ ) and the transformation $q \rightarrow \bar{q}$ to pass to the contragredient bimodules. With these labels one checks that the adjoint action of the U(1) factor is given by multiplication of the basis vectors $f$ by the following powers of $\lambda \in \mathrm{U}(1)$ :

$$
\begin{array}{ccccc} 
& e & \nu & u & d \\
f_{L} & -1 & -1 & \frac{1}{3} & \frac{1}{3} \\
f_{R} & -2 & 0 & \frac{4}{3} & -\frac{2}{3}
\end{array}
$$

\subsection{The classification of Dirac operators.}

To be precise we adopt the following,

Definition 2.6. A Dirac operator is a self-adjoint operator $D$ in $\mathcal{H}_{F}$ commuting with $J_{F}$, $\mathbb{C}_{F}=\{(\lambda, \lambda, 0)\} \in \mathcal{A}_{F}$, anticommuting with $\gamma_{F}$ and fulfilling the order one condition $\left[[D, a], b^{0}\right]=$ 0 for any $a, b \in \mathcal{A}_{F}$.

The physics meaning of the condition of commutation with $\mathbb{C}_{F}$ is to ensure that one gauge vector boson (the photon) remains massless.

In order to state the classification of Dirac operators we introduce the following notation, let $M_{e}, M_{\nu}, M_{d}, M_{u}$ and $M_{R}$ be three by three matrices, we then let $D(M)$ be the operator in $\mathcal{H}_{F}$ given by

$$
D(M)=\left[\begin{array}{cc}
S & T^{*} \\
T & \bar{S}
\end{array}\right]
$$

where

$$
S=S_{\ell} \oplus\left(S_{q} \otimes 1_{3}\right)
$$

and in the basis $\left(\nu_{R}, e_{R}, \nu_{L}, e_{L}\right)$ and $\left(u_{R}, d_{R}, u_{L}, d_{L}\right)$,

$$
S_{\ell}=\left[\begin{array}{cccc}
0 & 0 & M_{\nu}^{*} & 0 \\
0 & 0 & 0 & M_{e}^{*} \\
M_{\nu} & 0 & 0 & 0 \\
0 & M_{e} & 0 & 0
\end{array}\right] \quad S_{q}=\left[\begin{array}{cccc}
0 & 0 & M_{u}^{*} & 0 \\
0 & 0 & 0 & M_{d}^{*} \\
M_{u} & 0 & 0 & 0 \\
0 & M_{d} & 0 & 0
\end{array}\right]
$$

while the operator $T$ is 0 except on the subspace $\mathcal{H}_{\nu_{R}} \subset \mathcal{H}_{F}$ with basis the $\nu_{R}$ which it maps, using the matrix $M_{R}$, to the subspace $\mathcal{H}_{\bar{\nu}_{R}} \subset \mathcal{H}_{F}$ with basis the $\bar{\nu}_{R}$.

Theorem 2.7. (1) Let $D$ be a Dirac operator. There exists three by three matrices $M_{e}$, $M_{\nu}, M_{d}, M_{u}$ and $M_{R}$, with $M_{R}$ symmetric, such that $D=D(M)$.

(2) All operators $D(M)$ (with $M_{R}$ symmetric) are Dirac operators.

(3) The operators $D(M)$ and $D\left(M^{\prime}\right)$ are conjugate by a unitary operator commuting with $\mathcal{A}_{F}, \gamma_{F}$ and $J_{F}$ iff there exists unitary matrices $V_{j}$ and $W_{j}$ such that

$$
M_{e}^{\prime}=V_{1} M_{e} V_{3}^{*}, M_{\nu}^{\prime}=V_{2} M_{\nu} V_{3}^{*}, M_{d}^{\prime}=W_{1} M_{d} W_{3}^{*}, M_{u}^{\prime}=W_{2} M_{u} W_{3}^{*}, M_{R}^{\prime}=V_{2} M_{R} \bar{V}_{2}^{*}
$$

In particular Theorem 2.7 shows that the Dirac operators give all the required features, such as 
- Mixing matrices for quarks and leptons

- Unbroken color

- See-saw mechanism for right handed neutrinos

Let us briefly explain the last item, i.e. the analogue of the seesaw mechanism in our context. The restriction of $D(M)$ to the subspace of $\mathcal{H}_{F}$ with basis the $\left(\nu_{R}, \nu_{L}, \bar{\nu}_{R}, \bar{\nu}_{L}\right)$ is given by the matrix,

$$
\left[\begin{array}{cccc}
0 & M_{\nu}^{*} & M_{R}^{*} & 0 \\
M_{\nu} & 0 & 0 & 0 \\
M_{R} & 0 & 0 & \bar{M}_{\nu}^{*} \\
0 & 0 & \bar{M}_{\nu} & 0
\end{array}\right]
$$

Let us simplify to one generation and let $M_{R} \sim M$ be a very large mass term- the largest eigenvalue of $M_{R}$ will be set to the order of the unification scale by the equations of motion (41) of the spectral action below- while $M_{\nu} \sim m$ is much smaller 3 . The eigenvalues of the matrix (12) are then given by

$$
\frac{1}{2}\left( \pm M \pm \sqrt{M^{2}+4 m^{2}}\right)
$$

which gives two eigenvalues very close to $\pm M$ and two others very close to $\pm \frac{m^{2}}{M}$ as can be checked directly from the determinant of the matrix (12), which is equal to $\left|M_{\nu}\right|^{4} \sim m^{4}$ (for one generation).

\section{The spectral action for $M \times F$ And the Standard Model}

We now consider a 4-dimensional smooth compact Riemannian manifold $M$ with a fixed spin structure and recall that it is fully encoded by its Dirac spectral triple $\left(\mathcal{A}_{1}, \mathcal{H}_{1}, D_{1}\right)=$ $\left(C^{\infty}(M), L^{2}(M, S), \not_{M}\right)$. We then consider its product with the above finite geometry $\left(\mathcal{A}_{2}, \mathcal{H}_{2}, D_{2}\right)=\left(\mathcal{A}_{F}, \mathcal{H}_{F}, D_{F}\right)$. With $\left(\mathcal{A}_{j}, \mathcal{H}_{j}, D_{j}\right)$ of $K O$-dimensions 4 for $j=1$ and 6 for $j=2$, the product geometry is given by the rules,

$$
\mathcal{A}=\mathcal{A}_{1} \otimes \mathcal{A}_{2}, \mathcal{H}=\mathcal{H}_{1} \otimes \mathcal{H}_{2}, D=D_{1} \otimes 1+\gamma_{1} \otimes D_{2}, \gamma=\gamma_{1} \otimes \gamma_{2}, J=J_{1} \otimes J_{2}
$$

Note that it matters that $J_{1}$ commutes with $\gamma_{1}$ to check that $J$ commutes with $D$. The $K O$-dimension of the finite space $F$ is $6 \in \mathbb{Z} / 8$ and thus the $K O$-dimension of the product geometry $M \times F$ is now $2 \in \mathbb{Z} / 8$. In other words according to Appendix [7, definition 7.2 the commutation rules are

$$
J^{2}=-1, \quad J D=D J, \text { and } J \gamma=-\gamma J .
$$

Let us now explain how these rules allow to define a natural antisymmetric bilinear form on the even part $\mathcal{H}^{+}=\{\xi \in \mathcal{H}, \gamma \xi=\xi\}$ of $\mathcal{H}$.

Proposition 3.1. On a real spectral triple of $K O$-dimension $2 \in \mathbb{Z} / 8$, the following equality defines an antisymmetric bilinear form on $\mathcal{H}^{+}=\{\xi \in \mathcal{H}, \gamma \xi=\xi\}$,

$$
A_{D}\left(\xi^{\prime}, \xi\right)=\left\langle J \xi^{\prime}, D \xi\right\rangle, \quad \forall \xi, \xi^{\prime} \in \mathcal{H}^{+}
$$

The above trilinear pairing between $D, \xi$ and $\xi^{\prime}$ is gauge invariant under the adjoint action (cf. (48)) of the unitary group of $\mathcal{A}$,

$$
A_{D}\left(\xi^{\prime}, \xi\right)=A_{D_{u}}\left(\operatorname{Ad}(u) \xi^{\prime}, \operatorname{Ad}(u) \xi\right), \quad D_{u}=\operatorname{Ad}(u) D \operatorname{Ad}\left(u^{*}\right)
$$

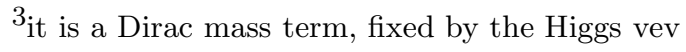


Now the Pfaffian of an antisymmetric bilinear form is best expressed in terms of the functional integral involving anticommuting "classical fermions" which at the formal level means that

$$
\operatorname{Pf}(A)=\int e^{-\frac{1}{2} A(\xi)} D[\xi]
$$

It is the use of the Pfaffian as a square root of the determinant that allows to solve the Fermion doubling puzzle which was pointed out in [15]. The solution obtained by a better choice of the $K O$-dimension of the space $F$ and hence of $M \times F$ is not unrelated to the point made in [11].

Theorem 3.2. Let $M$ be a Riemannian spin 4-manifold and $F$ the finite noncommutative geometry of KO-dimension 6 described above. Let $M \times F$ be endowed with the product metric.

(1) The unimodular subgroup of the unitary group acting by the adjoint representation $\operatorname{Ad}(u)$ in $\mathcal{H}$ is the group of gauge transformations of $S M$.

(2) The unimodular inner fluctuations A of the metric (cf. Appendix 7) are parameterized exactly by the gauge bosons of SM (including the Higgs doublet).

(3) The full standard model (see the explicit formula in \$5) minimally coupled with Einstein gravity is given in Euclidean form by the action functionat

$$
S=\operatorname{Tr}\left(f\left(D_{A} / \Lambda\right)\right)+\frac{1}{2}\left\langle J \xi, D_{A} \xi\right\rangle, \quad \xi \in \mathcal{H}^{+}
$$

applied to unimodular inner fluctuations $D_{A}=D+A+J A J^{-1}$ of the metric.

The proof is an excruciating computation, which is a variant of [2] (cf. [12] for a detailed version). After turning off gravity to simplify and working in flat space (after Wick rotation back to Lorentzian signature) one gets exactly the Lagrangian of \$5 which can hardly be fortuitous. The fermion doubling problem is resolved by the use of the Pfaffian, we checked that part for the Dirac mass terms, and trust that the same holds for the Majorana mass terms. There is one subtle point which is the use of the following chiral transformation:

$$
U=e^{i \frac{\pi}{4} \gamma_{5}}
$$

to transform the fermionic part of the action to the traditional one i.e. the Euclidean action for Fermi fields ( $c f .[5]$ ). While this transformation is innocent at the classical level, it is non-trivial at the quantum level and introduces some kind of Maslov index in the transition from our form of the Euclidean action to the more traditional one. We shall now give more details on the bosonic part of the action.

\section{Detailed form of the Bosonic aCtion}

We shall now give the precise form of the bosonic action, the calculation is entirely similar to [2] with new terms appearing from the presence of $M_{R}$.

\footnotetext{
${ }^{4}$ We take $f$ even and positive with $f^{(n)}(0)=0$ for $n \geq 1$ for definiteness. Note also that the components of $\xi$ anticommute so the antisymmetric form does not vanish.
} 
One lets $f_{k}=\int_{0}^{\infty} f(u) u^{k-1} d u$ for $k>0$ and $f_{0}=f(0)$. Also

$$
\begin{aligned}
a & =\operatorname{Tr}\left(M_{\nu}^{*} M_{\nu}+M_{e}^{*} M_{e}+3\left(M_{u}^{*} M_{u}+M_{d}^{*} M_{d}\right)\right) \\
b & =\operatorname{Tr}\left(\left(M_{\nu}^{*} M_{\nu}\right)^{2}+\left(M_{e}^{*} M_{e}\right)^{2}+3\left(M_{u}^{*} M_{u}\right)^{2}+3\left(M_{d}^{*} M_{d}\right)^{2}\right) \\
c & =\operatorname{Tr}\left(M_{R}^{*} M_{R}\right) \\
d & =\operatorname{Tr}\left(\left(M_{R}^{*} M_{R}\right)^{2}\right) \\
e & =\operatorname{Tr}\left(M_{R}^{*} M_{R} M_{\nu}^{*} M_{\nu}\right)
\end{aligned}
$$

The spectral action is given by a computation entirely similar to [2] which yields:

$$
\begin{aligned}
S & =\frac{1}{\pi^{2}}\left(48 f_{4} \Lambda^{4}-f_{2} \Lambda^{2} c+\frac{f_{0}}{4} d\right) \int \sqrt{g} d^{4} x \\
& +\frac{96 f_{2} \Lambda^{2}-f_{0} c}{24 \pi^{2}} \int R \sqrt{g} d^{4} x \\
& +\frac{f_{0}}{10 \pi^{2}} \int\left(\frac{11}{6} R^{*} R^{*}-3 C_{\mu \nu \rho \sigma} C^{\mu \nu \rho \sigma}\right) \sqrt{g} d^{4} x \\
& +\frac{\left(-2 a f_{2} \Lambda^{2}+e f_{0}\right)}{\pi^{2}} \int|\varphi|^{2} \sqrt{g} d^{4} x \\
& +\frac{f_{0}}{2 \pi^{2}} \int a\left|D_{\mu} \varphi\right|^{2} \sqrt{g} d^{4} x \\
& -\frac{f_{0}}{12 \pi^{2}} \int a R|\varphi|^{2} \sqrt{g} d^{4} x \\
& +\frac{f_{0}}{2 \pi^{2}} \int\left(g_{3}^{2} G_{\mu \nu}^{i} G^{\mu \nu i}+g_{2}^{2} F_{\mu \nu}^{\alpha} F^{\mu \nu \alpha}+\frac{5}{3} g_{1}^{2} B_{\mu \nu} B^{\mu \nu}\right) \sqrt{g} d^{4} x \\
& +\frac{f_{0}}{2 \pi^{2}} \int b|\varphi|^{4} \sqrt{g} d^{4} x
\end{aligned}
$$

where $(a, b, c, d, e)$ are defined above and $D_{\mu} \varphi$ is the minimal coupling. A simple change of variables as in [2], namely

$$
\mathbf{H}=\frac{\sqrt{a f_{0}}}{\pi} \varphi
$$

so that the kinetic term becomes

$$
\int \frac{1}{2}\left|D_{\mu} \mathbf{H}\right|^{2} \sqrt{g} d^{4} x
$$

and

$$
\frac{g_{3}^{2} f_{0}}{2 \pi^{2}}=\frac{1}{4}, \quad g_{3}^{2}=g_{2}^{2}=\frac{5}{3} g_{1}^{2}
$$

\footnotetext{
${ }^{5}$ here we differ slightly from [2] by a factor of $\sqrt{2}$ to match the conventions of Veltman [17]
} 
transforms the bosonic action into the form:

$$
\begin{aligned}
S & =\int d^{4} x \sqrt{g}\left[\frac{1}{2 \kappa_{0}^{2}} R+\alpha_{0} C_{\mu \nu \rho \sigma} C^{\mu \nu \rho \sigma}\right. \\
& +\gamma_{0}+\tau_{0}{ }^{*} R^{*} R+\delta_{0} R ;_{\mu}{ }^{\mu} \\
& +\frac{1}{4} G_{\mu \nu}^{i} G^{\mu \nu i}+\frac{1}{4} F_{\mu \nu}^{\alpha} F^{\mu \nu \alpha}+\frac{1}{4} B_{\mu \nu} B^{\mu \nu} \\
& \left.+\frac{1}{2}\left|D_{\mu} \mathbf{H}\right|^{2}-\mu_{0}^{2}|\mathbf{H}|^{2}-\frac{1}{12} R|\mathbf{H}|^{2}+\lambda_{0}|\mathbf{H}|^{4}\right]
\end{aligned}
$$

where

$$
\begin{aligned}
\frac{1}{\kappa_{0}^{2}} & =\frac{96 f_{2} \Lambda^{2}-f_{0} c}{12 \pi^{2}} \\
\mu_{0}^{2} & =2 \frac{f_{2} \Lambda^{2}}{f_{0}}-\frac{e}{a} \\
\alpha_{0} & =-\frac{3 f_{0}}{10 \pi^{2}} \\
\tau_{0} & =\frac{11 f_{0}}{60 \pi^{2}} \\
\delta_{0} & =-\frac{2}{3} \alpha_{0} \\
\gamma_{0} & =\frac{1}{\pi^{2}}\left(48 f_{4} \Lambda^{4}-f_{2} \Lambda^{2} c+\frac{f_{0}}{4} d\right) \\
\lambda_{0} & =\frac{\pi^{2}}{2 f_{0}} \frac{b}{a^{2}}=\frac{b g^{2}}{a^{2}}
\end{aligned}
$$

\section{Detailed form of the SPeCtral ACtion Without Gravity}

To make the comparison easier we Wick rotate back to Minkowski space and after turning off gravity by working in flat space (and addition of gauge fixing terms 6 ) the spectral action, after the change of variables summarized in table 1, is given by the following formula:

$$
\begin{gathered}
\mathcal{L}_{S M}=-\frac{1}{2} \partial_{\nu} g_{\mu}^{a} \partial_{\nu} g_{\mu}^{a}-g_{s} f^{a b c} \partial_{\mu} g_{\nu}^{a} g_{\mu}^{b} g_{\nu}^{c}-\frac{1}{4} g_{s}^{2} f^{a b c} f^{a d e} g_{\mu}^{b} g_{\nu}^{c} g_{\mu}^{d} g_{\nu}^{e}-\partial_{\nu} W_{\mu}^{+} \partial_{\nu} W_{\mu}^{-}- \\
M^{2} W_{\mu}^{+} W_{\mu}^{-}-\frac{1}{2} \partial_{\nu} Z_{\mu}^{0} \partial_{\nu} Z_{\mu}^{0}-\frac{1}{2 c_{w}^{2}} M^{2} Z_{\mu}^{0} Z_{\mu}^{0}-\frac{1}{2} \partial_{\mu} A_{\nu} \partial_{\mu} A_{\nu}-i g c_{w}\left(\partial_{\nu} Z_{\mu}^{0}\left(W_{\mu}^{+} W_{\nu}^{-}-W_{\nu}^{+} W_{\mu}^{-}\right)-\right. \\
\left.Z_{\nu}^{0}\left(W_{\mu}^{+} \partial_{\nu} W_{\mu}^{-}-W_{\mu}^{-} \partial_{\nu} W_{\mu}^{+}\right)+Z_{\mu}^{0}\left(W_{\nu}^{+} \partial_{\nu} W_{\mu}^{-}-W_{\nu}^{-} \partial_{\nu} W_{\mu}^{+}\right)\right)-i g s_{w}\left(\partial _ { \nu } A _ { \mu } \left(W_{\mu}^{+} W_{\nu}^{-}-\right.\right. \\
\left.\left.W_{\nu}^{+} W_{\mu}^{-}\right)-A_{\nu}\left(W_{\mu}^{+} \partial_{\nu} W_{\mu}^{-}-W_{\mu}^{-} \partial_{\nu} W_{\mu}^{+}\right)+A_{\mu}\left(W_{\nu}^{+} \partial_{\nu} W_{\mu}^{-}-W_{\nu}^{-} \partial_{\nu} W_{\mu}^{+}\right)\right)- \\
\frac{1}{2} g^{2} W_{\mu}^{+} W_{\mu}^{-} W_{\nu}^{+} W_{\nu}^{-}+\frac{1}{2} g^{2} W_{\mu}^{+} W_{\nu}^{-} W_{\mu}^{+} W_{\nu}^{-}+g^{2} c_{w}^{2}\left(Z_{\mu}^{0} W_{\mu}^{+} Z_{\nu}^{0} W_{\nu}^{-}-Z_{\mu}^{0} Z_{\mu}^{0} W_{\nu}^{+} W_{\nu}^{-}\right)+ \\
g^{2} s_{w}^{2}\left(A_{\mu} W_{\mu}^{+} A_{\nu} W_{\nu}^{-}-A_{\mu} A_{\mu} W_{\nu}^{+} W_{\nu}^{-}\right)+g^{2} s_{w} c_{w}\left(A_{\mu} Z_{\nu}^{0}\left(W_{\mu}^{+} W_{\nu}^{-}-W_{\nu}^{+} W_{\mu}^{-}\right)-\right. \\
\left.2 A_{\mu} Z_{\mu}^{0} W_{\nu}^{+} W_{\nu}^{-}\right)-\frac{1}{2} \partial_{\mu} H \partial_{\mu} H-2 M^{2} \alpha_{h} H^{2}-\partial_{\mu} \phi^{+} \partial_{\mu} \phi^{-}-\frac{1}{2} \partial_{\mu} \phi^{0} \partial_{\mu} \phi^{0}- \\
\beta_{h}\left(\frac{2 M^{2}}{g^{2}}+\frac{2 M}{g} H+\frac{1}{2}\left(H^{2}+\phi^{0} \phi^{0}+2 \phi^{+} \phi^{-}\right)\right)+\frac{2 M^{4}}{g^{2}} \alpha_{h}-g \alpha_{h} M\left(H^{3}+H \phi^{0} \phi^{0}+2 H \phi^{+} \phi^{-}\right)- \\
\frac{1}{8} g^{2} \alpha_{h}\left(H^{4}+\left(\phi^{0}\right)^{4}+4\left(\phi^{+} \phi^{-}\right)^{2}+4\left(\phi^{0}\right)^{2} \phi^{+} \phi^{-}+4 H^{2} \phi^{+} \phi^{-}+2\left(\phi^{0}\right)^{2} H^{2}\right)-g M W_{\mu}^{+} W_{\mu}^{-} H- \\
\frac{1}{2} g \frac{M}{c_{w}^{2}} Z_{\mu}^{0} Z_{\mu}^{0} H-\frac{1}{2} i g\left(W_{\mu}^{+}\left(\phi^{0} \partial_{\mu} \phi^{-}-\phi^{-} \partial_{\mu} \phi^{0}\right)-W_{\mu}^{-}\left(\phi^{0} \partial_{\mu} \phi^{+}-\phi^{+} \partial_{\mu} \phi^{0}\right)\right)+ \\
\frac{1}{2} g\left(W_{\mu}^{+}\left(H \partial_{\mu} \phi^{-}-\phi^{-} \partial_{\mu} H\right)+W_{\mu}^{-}\left(H \partial_{\mu} \phi^{+}-\phi^{+} \partial_{\mu} H\right)\right)+\frac{1}{2} g \frac{1}{c_{w}}\left(Z_{\mu}^{0}\left(H \partial_{\mu} \phi^{0}-\phi^{0} \partial_{\mu} H\right)+\right. \\
M\left(\frac{1}{c_{w}} Z_{\mu}^{0} \partial_{\mu} \phi^{0}+W_{\mu}^{+} \partial_{\mu} \phi^{-}+W_{\mu}^{-} \partial_{\mu} \phi^{+}\right)-i g \frac{s_{w}^{2}}{c_{w}} Z_{\mu}^{0}\left(W_{\mu}^{+} \phi^{-}-W_{\mu}^{-} \phi^{+}\right)+i g s_{w} M A_{\mu}\left(W_{\mu}^{+} \phi^{-}-\right.
\end{gathered}
$$

\footnotetext{
${ }^{6}$ We add the Feynman gauge fixing terms just to simplify the form of the gauge kinetic terms
} 


$$
\begin{aligned}
& \left.W_{\mu}^{-} \phi^{+}\right)-i g \frac{1-2 c_{w}^{2}}{2 c_{w}} Z_{\mu}^{0}\left(\phi^{+} \partial_{\mu} \phi^{-}-\phi^{-} \partial_{\mu} \phi^{+}\right)+i g s_{w} A_{\mu}\left(\phi^{+} \partial_{\mu} \phi^{-}-\phi^{-} \partial_{\mu} \phi^{+}\right)- \\
& \frac{1}{4} g^{2} W_{\mu}^{+} W_{\mu}^{-}\left(H^{2}+\left(\phi^{0}\right)^{2}+2 \phi^{+} \phi^{-}\right)-\frac{1}{8} g^{2} \frac{1}{c_{w}^{2}} Z_{\mu}^{0} Z_{\mu}^{0}\left(H^{2}+\left(\phi^{0}\right)^{2}+2\left(2 s_{w}^{2}-1\right)^{2} \phi^{+} \phi^{-}\right)- \\
& \frac{1}{2} g^{2} \frac{s_{w}^{2}}{c_{w}} Z_{\mu}^{0} \phi^{0}\left(W_{\mu}^{+} \phi^{-}+W_{\mu}^{-} \phi^{+}\right)-\frac{1}{2} i g^{2} \frac{s_{w}^{2}}{c_{w}} Z_{\mu}^{0} H\left(W_{\mu}^{+} \phi^{-}-W_{\mu}^{-} \phi^{+}\right)+\frac{1}{2} g^{2} s_{w} A_{\mu} \phi^{0}\left(W_{\mu}^{+} \phi^{-}+\right. \\
& \left.W_{\mu}^{-} \phi^{+}\right)+\frac{1}{2} i g^{2} s_{w} A_{\mu} H\left(W_{\mu}^{+} \phi^{-}-W_{\mu}^{-} \phi^{+}\right)-g^{2} \frac{s_{w}}{c_{w}}\left(2 c_{w}^{2}-1\right) Z_{\mu}^{0} A_{\mu} \phi^{+} \phi^{-}-g^{2} s_{w}^{2} A_{\mu} A_{\mu} \phi^{+} \phi^{-}+ \\
& \frac{1}{2} i g_{s} \lambda_{i j}^{a}\left(\bar{q}_{i}^{\sigma} \gamma^{\mu} q_{j}^{\sigma}\right) g_{\mu}^{a}-\bar{e}^{\lambda}\left(\gamma \partial+m_{e}^{\lambda}\right) e^{\lambda}-\bar{\nu}^{\lambda}\left(\gamma \partial+m_{\nu}^{\lambda}\right) \nu^{\lambda}-\bar{u}_{j}^{\lambda}\left(\gamma \partial+m_{u}^{\lambda}\right) u_{j}^{\lambda}-\bar{d}_{j}^{\lambda}\left(\gamma \partial+m_{d}^{\lambda}\right) d_{j}^{\lambda}+ \\
& i g s_{w} A_{\mu}\left(-\left(\bar{e}^{\lambda} \gamma^{\mu} e^{\lambda}\right)+\frac{2}{3}\left(\bar{u}_{j}^{\lambda} \gamma^{\mu} u_{j}^{\lambda}\right)-\frac{1}{3}\left(\bar{d}_{j}^{\lambda} \gamma^{\mu} d_{j}^{\lambda}\right)\right)+\frac{i g}{4 c_{w}} Z_{\mu}^{0}\left\{\left(\bar{\nu}^{\lambda} \gamma^{\mu}\left(1+\gamma^{5}\right) \nu^{\lambda}\right)+\left(\overline { e } ^ { \lambda } \gamma ^ { \mu } \left(4 s_{w}^{2}-\right.\right.\right. \\
& \left.\left.\left.1-\gamma^{5}\right) e^{\lambda}\right)+\left(\bar{d}_{j}^{\lambda} \gamma^{\mu}\left(\frac{4}{3} s_{w}^{2}-1-\gamma^{5}\right) d_{j}^{\lambda}\right)+\left(\bar{u}_{j}^{\lambda} \gamma^{\mu}\left(1-\frac{8}{3} s_{w}^{2}+\gamma^{5}\right) u_{j}^{\lambda}\right)\right\}+ \\
& \frac{i g}{2 \sqrt{2}} W_{\mu}^{+}\left(\left(\bar{\nu}^{\lambda} \gamma^{\mu}\left(1+\gamma^{5}\right) U_{\lambda \kappa}^{l e p} e^{\kappa}\right)+\left(\bar{u}_{j}^{\lambda} \gamma^{\mu}\left(1+\gamma^{5}\right) C_{\lambda \kappa} d_{j}^{\kappa}\right)\right)+ \\
& \frac{i g}{2 \sqrt{2}} W_{\mu}^{-}\left(\left(\bar{e}^{\kappa} U_{\kappa \lambda}^{l e p^{\dagger}} \gamma^{\mu}\left(1+\gamma^{5}\right) \nu^{\lambda}\right)+\left(\bar{d}_{j}^{\kappa} C_{\kappa \lambda}^{\dagger} \gamma^{\mu}\left(1+\gamma^{5}\right) u_{j}^{\lambda}\right)\right)+ \\
& \frac{i g}{2 M \sqrt{2}} \phi^{+}\left(-m_{e}^{\kappa}\left(\bar{\nu}^{\lambda} U_{\lambda \kappa}^{l e p}\left(1-\gamma^{5}\right) e^{\kappa}\right)+m_{\nu}^{\lambda}\left(\bar{\nu}^{\lambda} U_{\lambda \kappa}^{l e p}\left(1+\gamma^{5}\right) e^{\kappa}\right)+\right. \\
& \frac{i g}{2 M \sqrt{2}} \phi^{-}\left(m_{e}^{\lambda}\left(\bar{e}^{\lambda} U^{l e p^{\dagger}}{ }_{\lambda \kappa}\left(1+\gamma^{5}\right) \nu^{\kappa}\right)-m_{\nu}^{\kappa}\left(\bar{e}^{\lambda} U^{l e p_{\lambda \kappa}^{\dagger}}\left(1-\gamma^{5}\right) \nu^{\kappa}\right)-\frac{g}{2} \frac{m_{\nu}^{\lambda}}{M} H\left(\bar{\nu}^{\lambda} \nu^{\lambda}\right)-\right. \\
& \frac{g}{2} \frac{m_{e}^{\lambda}}{M} H\left(\bar{e}^{\lambda} e^{\lambda}\right)+\frac{i g}{2} \frac{m_{\nu}^{\lambda}}{M} \phi^{0}\left(\bar{\nu}^{\lambda} \gamma^{5} \nu^{\lambda}\right)-\frac{i g}{2} \frac{m_{e}^{\lambda}}{M} \phi^{0}\left(\bar{e}^{\lambda} \gamma^{5} e^{\lambda}\right)-\frac{1}{4} \bar{\nu}_{\lambda} M_{\lambda \kappa}^{R}\left(1-\gamma_{5}\right) \hat{\nu}_{\kappa}- \\
& \frac{1}{4} \overline{\bar{\nu}_{\lambda} M_{\lambda \kappa}^{R}\left(1-\gamma_{5}\right) \hat{\nu}_{\kappa}}+\frac{i g}{2 M \sqrt{2}} \phi^{+}\left(-m_{d}^{\kappa}\left(\bar{u}_{j}^{\lambda} C_{\lambda \kappa}\left(1-\gamma^{5}\right) d_{j}^{\kappa}\right)+m_{u}^{\lambda}\left(\bar{u}_{j}^{\lambda} C_{\lambda \kappa}\left(1+\gamma^{5}\right) d_{j}^{\kappa}\right)+\right. \\
& \frac{i g}{2 M \sqrt{2}} \phi^{-}\left(m_{d}^{\lambda}\left(\bar{d}_{j}^{\lambda} C_{\lambda \kappa}^{\dagger}\left(1+\gamma^{5}\right) u_{j}^{\kappa}\right)-m_{u}^{\kappa}\left(\bar{d}_{j}^{\lambda} C_{\lambda \kappa}^{\dagger}\left(1-\gamma^{5}\right) u_{j}^{\kappa}\right)-\frac{g}{2} \frac{m_{u}^{\lambda}}{M} H\left(\bar{u}_{j}^{\lambda} u_{j}^{\lambda}\right)-\frac{g}{2} \frac{m_{d}^{\lambda}}{M} H\left(\bar{d}_{j}^{\lambda} d_{j}^{\lambda}\right)+\right. \\
& \frac{i g}{2} \frac{m_{u}^{\lambda}}{M} \phi^{0}\left(\bar{u}_{j}^{\lambda} \gamma^{5} u_{j}^{\lambda}\right)-\frac{i g}{2} \frac{m_{d}^{\lambda}}{M} \phi^{0}\left(\bar{d}_{j}^{\lambda} \gamma^{5} d_{j}^{\lambda}\right)
\end{aligned}
$$

This formula compares nicely with [17]. Besides the addition of the neutrino mass terms, and absence of the ghost terms there is only one difference: in the spectral action Lagrangian one gets the term:

$$
M\left(\frac{1}{c_{w}} Z_{\mu}^{0} \partial_{\mu} \phi^{0}+W_{\mu}^{+} \partial_{\mu} \phi^{-}+W_{\mu}^{-} \partial_{\mu} \phi^{+}\right)
$$

while in the Veltman's formula [17] one gets instead the following:

$$
-M^{2} \phi^{+} \phi^{-}-\frac{1}{2 c_{w}^{2}} M^{2} \phi^{0} \phi^{0}
$$

This difference comes from the gauge fixing term

$$
\mathcal{L}_{\text {fix }}=-\frac{1}{2} \mathcal{C}^{2}, \quad \mathcal{C}_{a}=-\partial_{\mu} W_{a}^{\mu}+M_{a} \phi_{a}
$$

given by the Feynman-t'Hooft gauge in Veltman's formula [17, indeed one has

$$
\mathcal{L}_{f i x}=-\frac{1}{2}\left(\partial_{\mu} W_{a}^{\mu}\right)^{2}-\frac{1}{2 c_{w}^{2}} M^{2} \phi^{0} \phi^{0}-M^{2} \phi^{+} \phi^{-}+M\left(\frac{1}{c_{w}} \phi^{0} \partial_{\mu} Z_{\mu}^{0}+\phi^{-} \partial_{\mu} W_{\mu}^{+}+\phi^{+} \partial_{\mu} W_{\mu}^{-}\right)
$$

The numerical values are similar to those of 2 and in particular one gets the same value of gauge couplings as in grand unified SU(5)-theory. This means that in the above formula the values of $g, g_{s}$ and $s_{w}, c_{w}$ are fixed exactly as in 2] at

$$
g_{s}=g, \quad \operatorname{tg}(w)^{2}=\frac{3}{5}
$$

One also gets a specific value of the Higgs scattering parameter $\alpha_{h}$, as in [2] (which agrees with [13]),

$$
\alpha_{h}=\frac{8 b}{a^{2}}
$$


(with the notations (16)) which is of the order of $\frac{8}{3}$ if there is a dominating top mass. The change of notations for the Higgs fields is

$$
\mathbf{H}=\frac{1}{\sqrt{2}} \frac{\sqrt{a}}{g}(1+\psi)=\left(\frac{2 M}{g}+H-i \phi^{0},-i \sqrt{2} \phi^{+}\right),
$$

while the huge term in $\Lambda^{2}$ in the spectral action can be absorbed by a suitable choice of the tadpole constant $\beta_{h}$

$$
\beta_{h}=2 \alpha_{h} M^{2}-4 \frac{f_{2} \Lambda^{2}}{f_{0}}+2 \frac{e}{a}
$$

Note that the matrices $M_{u}, M_{d}, M_{\nu}$ and $M_{e}$ are only relevant up to an overall scale. Indeed they only enter in the coupling of the Higgs with fermions and because of the rescaling (18) only by the terms

$$
k_{x}=\frac{\pi}{\sqrt{a f_{0}}} M_{x}, \quad x \in\{u, d, \nu, e\}
$$

which are dimensionless matrices by construction. The conversion for the mass matrices is

$$
\begin{aligned}
\left(k_{u}\right)_{\lambda \kappa} & =\frac{g}{2 M} m_{u}^{\lambda} \delta_{\lambda}^{\kappa} \\
\left(k_{d}\right)_{\lambda \kappa} & =\frac{g}{2 M} m_{d}^{\mu} C_{\lambda \mu} \delta_{\mu}^{\rho} C_{\rho \kappa}^{\dagger} \\
\left(k_{\nu}\right)_{\lambda \kappa} & =\frac{g}{2 M} m_{\nu}^{\lambda} \delta_{\lambda}^{\kappa} \\
\left(k_{e}\right)_{\lambda \kappa} & =\frac{g}{2 M} m_{e}^{\mu} U_{\lambda \mu}^{l e p} \delta_{\mu}^{\rho} U_{\rho \kappa}^{l e p}{ }_{\rho \kappa}^{\dagger}
\end{aligned}
$$

It might seem at first sight that one can simply use (37) to define the matrices $k_{x}$ but this overlooks the fact that (36) implies one constraint:

$$
\operatorname{Tr}\left(k_{\nu}^{*} k_{\nu}+k_{e}^{*} k_{e}+3\left(k_{u}^{*} k_{u}+k_{d}^{*} k_{d}\right)\right)=2 g^{2},
$$

using (19) to replace $\frac{\pi^{2}}{f_{0}}$ by $2 g^{2}$. When expressed in the right hand side i.e. the standard model parameters this gives

$$
\sum_{\lambda}\left(m_{\nu}^{\lambda}\right)^{2}+\left(m_{e}^{\lambda}\right)^{2}+3\left(m_{u}^{\lambda}\right)^{2}+3\left(m_{d}^{\lambda}\right)^{2}=8 M^{2}
$$

where $M$ is the mass of the $W$ boson. Thus with the standard notation ([13]) for the Yukawa couplings, so that the fermion masses are $m_{f}=\frac{1}{\sqrt{2}} y_{f} v, v=\frac{2 M}{g}$ the relation reads

$$
\sum_{\lambda}\left(y_{\nu}^{\lambda}\right)^{2}+\left(y_{e}^{\lambda}\right)^{2}+3\left(y_{u}^{\lambda}\right)^{2}+3\left(y_{d}^{\lambda}\right)^{2}=4 g^{2}
$$

Neglecting the other Yukawa coupling except for the top quark, and imposing the relation (40) at unification scale, then running it downwards using the renormalization group one gets the boundary value $\frac{2}{\sqrt{3}} g \sim 0.597$ for $y_{t}$ at unification scale which gives a Fermi scale value of the order of $y_{0}=\sim 1.102$ and a top quark mass of the order of $\frac{1}{\sqrt{2}} y_{0} v \sim 173 y_{0} \mathrm{GeV}$. This is fine since a large neglected tau neutrino Yukawa coupling (allowed by the see-saw mechanism) similar to that of the top quark, lowers the value at unification by a factor of $\sqrt{\frac{3}{4}}$ which has the effect of lowering the value of $y_{0}$ to $y_{0} \sim 1.04$. This yields an acceptable value for the top quark mass (whose Yukawa coupling is $y_{0} \sim 1$ ), given that we still neglected all other smaller Yukawa couplings. 


\begin{tabular}{|c|c|c|c|}
\hline Standard Model & notation & notation & Spectral Action \\
\hline Higgs Boson & $\varphi=\left(\frac{2 M}{g}+H-i \phi^{0},-i \sqrt{2} \phi^{+}\right)$ & $\mathbf{H}=\frac{1}{\sqrt{2}} \frac{\sqrt{a}}{g}(1+\psi)$ & Inner metric ${ }^{(0,1)}$ \\
\hline Gauge bosons & $A_{\mu}, Z_{\mu}^{0}, W_{\mu}^{ \pm}, g_{\mu}^{a}$ & $(B, W, V)$ & Inner metric ${ }^{(1,0)}$ \\
\hline $\begin{array}{c}\text { Fermion masses } \\
u, \nu\end{array}$ & $m_{u}, m_{\nu}$ & $M_{u}=\delta_{u}, M_{\nu}=\delta_{\nu}$ & $\operatorname{Dirac}^{(0,1)}$ in $u, \nu$ \\
\hline $\begin{array}{l}\text { CKM matrix } \\
\text { Masses down }\end{array}$ & $C_{\lambda}^{\kappa}, m_{d}$ & $M_{d}=C \delta_{d} C^{\dagger}$ & $\operatorname{Dirac}^{(0,1)}$ in $d$ \\
\hline $\begin{array}{l}\text { Lepton mixing } \\
\text { Masses leptons } e\end{array}$ & $U_{\lambda \kappa}^{l e p}, m_{e}$ & $M_{e}=U^{l e p} \delta_{e} U^{l e p} \dagger$ & $\operatorname{Dirac}^{(0,1)}$ in $e$ \\
\hline $\begin{array}{c}\text { Majorana } \\
\text { mass matrix }\end{array}$ & $M^{R}$ & $M_{R}$ & $\operatorname{Dirac}^{(0,1)}$ in $\nu_{R}, \bar{\nu}_{R}$ \\
\hline Gauge couplings & $g_{1}=g \operatorname{tg}(w), g_{2}=g, g_{3}=g_{s}$ & $g_{3}^{2}=g_{2}^{2}=\frac{5}{3} g_{1}^{2}$ & $\begin{array}{c}\text { Fixed at } \\
\text { unification }\end{array}$ \\
\hline $\begin{array}{c}\text { Higgs scattering } \\
\text { parameter }\end{array}$ & $\frac{1}{8} g^{2} \alpha_{h}, \alpha_{h}=\frac{m_{h}^{2}}{4 M^{2}}$ & $\lambda_{0}=g^{2} \frac{b}{a^{2}}$ & $\begin{array}{c}\text { Fixed at } \\
\text { unification }\end{array}$ \\
\hline Tadpole constant & $\beta_{h},\left(-\alpha_{h} M^{2}+\frac{\beta_{h}}{2}\right)|\varphi|^{2}$ & $\mu_{0}^{2}=2 \frac{f_{2} \Lambda^{2}}{f_{0}}-\frac{e}{a}$ & $-\mu_{0}^{2}|\mathbf{H}|^{2}$ \\
\hline Graviton & $g_{\mu \nu}$ & $\not \partial_{M}$ & $\operatorname{Dirac}^{(1,0)}$ \\
\hline
\end{tabular}

TABle 1. Conversion from Spectral Action to Standard Model

The conversion table 1 shows that all the mass parameters of the standard model now acquire geometric meaning as components of the noncommutative metric as displayed in the right column.

\section{INTERPRETATION}

It is not clear what the physics meaning is since unlike in grand unified theories one is still lacking a renormalizable theory that would take over above the unification energy. But one can nevertheless hope that such a theory will be discovered along the lines of QFT on 
noncommutative spaces, or even that the fundamental theory has selected a preferred scale and is a fully unified theory at the operator theoretic level (i.e. a kind of spectral random matrix theory where the operator $D$ varies in the symplectic ensemble corresponding to the commutation with $i=\sqrt{-1}$ and $J$ that generate the quaternions) of which the standard model coupled with gravity is just a manifestation when one integrates the high energy modes á la Wilson. Then following [2] one can take the value of the Higgs quartic self-coupling (33) as an indication at that same energy and ([13]) get a rough estimate (around $170 \mathrm{GeV}$ ) for the Higgs mass under the "big desert" hypothesis. It is satisfactory that the prediction for the Weinberg angle (the same as $\mathrm{SU}(5)$ GUT) is not too far off and that the mass relation gives a sensible answer. But it is of course very likely that instead of the big desert one will meet gradual refinements of the noncommutative geometry $M \times F$ when climbing in energy to the unification scale.

The naturalness problem is of course still there, but interestingly the new terms involving $M_{R}$ provide room for obtaining in the spectral action a term that mimics the nasty quadratic divergence, whose coefficient changes sign under the running of the remormalization group. This freedom holds provided that the number of generations is $>1$. The quadratic coupling is $\mu_{0}^{2}=2 \frac{f_{2} \Lambda^{2}}{f_{0}}-\frac{e}{a}$. The presence of the new term $-\frac{e}{a}$ (which was absent in [2]) allows for the possibility that the sign of this mass term is arbitrary provided there are at least two generations. We shall assume to discuss this point that the matrix $M_{R}$ is a multiple of a fixed matrix $k_{R}$ i.e. is of the form $M_{R}=x k_{R}$. The value of $x$ is fixed by the equations of motion of the spectral action i.e. by minimizing the cosmological term. It gives

$$
x^{2}=\frac{2 f_{2} \Lambda^{2} \operatorname{Tr}\left(k_{R}^{*} k_{R}\right)}{f_{0} \operatorname{Tr}\left(\left(k_{R}^{*} k_{R}\right)^{2}\right)}, \quad M_{R}^{*} M_{R}=\frac{2 f_{2} \Lambda^{2}}{f_{0}} \frac{k_{R}^{*} k_{R} \operatorname{Tr}\left(k_{R}^{*} k_{R}\right)}{\operatorname{Tr}\left(\left(k_{R}^{*} k_{R}\right)^{2}\right)}
$$

Using (41) and (36) one gets

$$
\mu_{0}^{2}=2 \Lambda^{2} \frac{f_{2}}{f_{0}}(1-X), \quad X=\frac{\operatorname{Tr}\left(k_{R}^{*} k_{R} k_{\nu}^{*} k_{\nu}\right) \operatorname{Tr}\left(k_{R}^{*} k_{R}\right)}{\operatorname{Tr}\left(k_{\nu}^{*} k_{\nu}+k_{e}^{*} k_{e}+3\left(k_{u}^{*} k_{u}+k_{d}^{*} k_{d}\right)\right) \operatorname{Tr}\left(\left(k_{R}^{*} k_{R}\right)^{2}\right)}
$$

In order to compare $X$ with 1 we need to determine the range of variation of the largest eigenvalue of $\rho\left(k_{R}\right)=\frac{k_{R}^{*} k_{R} \operatorname{Tr}\left(k_{R}^{*} k_{R}\right)}{\operatorname{Tr}\left(\left(k_{R}^{*} k_{R}\right)^{2}\right)}$ as a function of the number $N$ of generations. One finds that this range of variation, for $k_{R} \in M_{N}(\mathbb{C})$, is the interval

$$
\left[1, \frac{1}{2}(1+\sqrt{N})\right]
$$

This suffices to show that provided the number $N$ of generations is $>1$, there is room to get a small value of $\mu_{0}^{2}$. Note that a similar discussion applies to the cosmological term $\gamma_{0}$ which inherits a negative contribution from the presence of the $M_{R}$ term.

\section{Appendix: Real Structure and inner fluctuations}

We just briefly recall here the definition of spectral triple $(\mathcal{A}, \mathcal{H}, D)$ and of real structure [7]:

Definition 7.1. A spectral triple $(\mathcal{A}, \mathcal{H}, D)$ is given by an involutive unital algebra $\mathcal{A}$ represented as operators in a Hilbert space $\mathcal{H}$ and a self-adjoint operator $D$ with compact resolvent such that all commutators $[D, a]$ are bounded for $a \in \mathcal{A}$.

A spectral triple is even if the Hilbert space $\mathcal{H}$ is endowed with a $\mathbb{Z} / 2$ - grading $\gamma$ which commutes with any $a \in \mathcal{A}$ and anticommutes with $D$. 
Definition 7.2. A real structure of $K O$-dimension $n \in \mathbb{Z} / 8$ on a spectral triple $(\mathcal{A}, \mathcal{H}, D)$ is an antilinear isometry $J: \mathcal{H} \rightarrow \mathcal{H}$, with the property that

$$
J^{2}=\varepsilon, \quad J D=\varepsilon^{\prime} D J, \text { and } J \gamma=\varepsilon^{\prime \prime} \gamma J \text { (even case). }
$$

The numbers $\varepsilon, \varepsilon^{\prime}, \varepsilon^{\prime \prime} \in\{-1,1\}$ are a function of $n \bmod 8$ given by

\begin{tabular}{|c|rrrrrrrr|}
\hline $\mathbf{n}$ & 0 & 1 & 2 & 3 & 4 & 5 & 6 & 7 \\
\hline \hline$\varepsilon$ & 1 & 1 & -1 & -1 & -1 & -1 & 1 & 1 \\
$\varepsilon^{\prime}$ & 1 & -1 & 1 & 1 & 1 & -1 & 1 & 1 \\
$\varepsilon^{\prime \prime}$ & 1 & & -1 & & 1 & & -1 & \\
\hline
\end{tabular}

Moreover, the action of $\mathcal{A}$ satisfies the commutation rule

$$
\left[a, b^{0}\right]=0 \quad \forall a, b \in \mathcal{A},
$$

where

$$
b^{0}=J b^{*} J^{-1} \quad \forall b \in \mathcal{A},
$$

and the operator $D$ satisfies

$$
\left[[D, a], b^{0}\right]=0 \quad \forall a, b \in \mathcal{A} .
$$

The key role of the real structure $J$ is to yield the following adjoint action of the unitary group $\mathcal{U}$ of the algebra $\mathcal{A}$ on the hilbert space $\mathcal{H}$ (of spinors). One defines a right $\mathcal{A}$-module structure on $\mathcal{H}$ by

$$
\xi b=b^{0} \xi, \quad \forall \xi \in \mathcal{H}, \quad b \in \mathcal{A}
$$

The unitary group of the algebra $\mathcal{A}$ then acts by the "adjoint representation" in $\mathcal{H}$ in the form

$$
\xi \in \mathcal{H} \rightarrow \operatorname{Ad}(u) \xi=u \xi u^{*}, \quad \forall \xi \in \mathcal{H}, \quad u \in \mathcal{A}, \quad u u^{*}=u^{*} u=1,
$$

and the inner fluctuation of the metric is given by

$$
D \rightarrow D_{A}=D+A+\varepsilon^{\prime} J A J^{-1}
$$

where $A$ is a self-adjoint operator of the form

$$
A=\sum a_{j}\left[D, b_{j}\right], \quad a_{j}, b_{j} \in \mathcal{A} .
$$

The unimodular inner fluctuations are obtained by restricting to those $A$ which are traceless i.e. fulfill the condition $\operatorname{Tr}(A)=0$.

\section{AKNOWLEDGEMENTS}

The detailed computations and extension of this work to the left-right model will appear in a joint work with Ali Chamseddine and Matilde Marcolli. The need to have independence between the $\mathrm{KO}$-dimension and the metric dimension already emerged implicitly in the work of L. Dąbrowski and A. Sitarz on Podleś quantum spheres [10. The results of this work were announced in a talk at the Newton Institute in July 2006, and the fear of a numerical error in the above computations delayed the present publication. It is a pleasure to acknowledge the independent preprint by John Barrett (A Lorentzian version of the non-commutative geometry of the standard model of particle physics) with a similar solution of the fermion doubling problem which accelerated the present publication. 


\section{REFERENCES}

[1] A. Chamseddine, A. Connes, Universal Formula for Noncommutative Geometry Actions: Unification of Gravity and the Standard Model, Phys. Rev. Lett. 77, 486804871 (1996).

[2] A. Chamseddine, A. Connes, The Spectral Action Principle, Comm. Math. Phys. 186, 731-750 (1997).

[3] A. Chamseddine, A. Connes, Scale Invariance in the Spectral Action, hep-th/0512169 to appear in Jour. Math. Phys

[4] A. Chamseddine, A. Connes, Inner fluctuations of the spectral action, hep-th/0605011.

[5] S. Coleman, Aspects of symmetry, Selected Erice Lectures, Cambridge University Press, 1985.

[6] A. Connes, Noncommutative geometry, Academic Press (1994).

[7] A. Connes, Non commutative geometry and reality, Journal of Math. Physics 36 no. 11 (1995).

[8] A. Connes, Gravity coupled with matter and the foundation of noncommutative geometry, Comm. Math. Phys. (1995)

[9] A. Connes, M. Marcolli Quantum fileds, noncommutative spaces and motives, Book in preparation.

[10] L. Dąbrowski, A. Sitarz, Dirac operator on the standard Podleś quantum sphere, Noncommutative Geometry and Quantum Groups, Banach Centre Publications 61, Hajac, P. M. and Pusz, W. (eds.), Warszawa: IMPAN, 2003, pp. 49-58.

[11] J. Gracia-Bonda, B. Iochum, T. Schucker, The standard model in noncommutative geometry and fermion doubling. Phys. Lett. B 416 no. 1-2 (1998), 123-128.

[12] D. Kastler, Noncommutative geometry and fundamental physical interactions: The Lagrangian level, Journal. Math. Phys. 41 (2000), 3867-3891.

[13] M. Knecht, T. Schucker Spectral action and big desert hep-ph/065166

[14] O. Lauscher, M. Reuter, Asymptotic Safety in Quantum Einstein Gravity: nonperturbative renormalizability and fractal spacetime structure, hep-th/0511260

[15] F. Lizzi, G. Mangano, G. Miele, G. Sparano, Fermion Hilbert space and Fermion Doubling in the Noncommutative Geometry Approach to Gauge Theories hep-th/9610035.

[16] R.N. Mohapatra, P.B. Pal, Massive neutrinos in physics and astrophysics, World Scientific, 2004.

[17] M. Veltman, Diagrammatica: the path to Feynman diagrams, Cambridge Univ. Press, 1994. 